\title{
BMJ Open Reflections on communication of disease prognosis and life expectancy by patients with colorectal cancer undergoing palliative care: a qualitative study
}

\author{
Gudrun Rohde, ${ }^{1,2}$ Ulrika Söderhamn, ${ }^{3}$ Ingvild Vistad ${ }^{4}$
}

To cite: Rohde G, Söderhamn U, Vistad I. Reflections on communication of disease prognosis and life expectancy by patients with colorectal cancer undergoing palliative care: a qualitative study. BMJ Open 2019;9:e023463. doi:10.1136/ bmjopen-2018-023463

- Prepublication history for this paper is available online. To view these files, please visit the journal online (http://dx.doi org/10.1136/bmjopen-2018023463).

Received 10 April 2018 Revised 23 December 2018 Accepted 7 February 2019

\section{Check for updates}

(c) Author(s) (or their employer(s)) 2019. Re-use permitted under CC BY-NC. No commercial re-use. See rights and permissions. Published by BMJ.

${ }^{1}$ Faculty of Health and Sport Sciences, University of Agder, Kristiansand, Norway ${ }^{2}$ Department of Clinical Research, Sorlandet Hospital, Kristiansand, Norway ${ }^{3}$ Department of Health and Nursing Sciences, Faculty of Health and Sport Sciences, University of Agder, Centre for Caring Research-Southern Norway, Grimstad, Norway ${ }^{4}$ Department of Obstetric and Gynaecology, Sorlandet Hospital Kristiansand, Kristiansand, Norway

Correspondence to Dr Gudrun Rohde; gudrun.e.rohde@uia.no

\section{ABSTRACT}

Objectives Patients with colorectal cancer undergoing palliative treatment receive extensive treatment-related information throughout their disease trajectory. We aimed to explore the experiences of patients with incurable colorectal cancer while in palliative care and their reflections on the information provided by physicians and nurses. Our main focus was the patients' thoughts about how information about disease status and life expectancy was communicated, from the first time that they were informed about the incurable nature of their disease through to postsurgery palliative treatment.

Settings Patients with colorectal cancer receiving palliative chemotherapy.

Research design We used a qualitative approach, and the data were analysed by qualitative content analysis. Participants 20 patients (34-75 years of age) were included in the study; 12 received first-line chemotherapy and 8 received second-line chemotherapy. Eleven patients were treated by oncologists, and nine were treated by junior physicians.

Results Data-driven empirical analysis identified three themes: (1) inadequate information during the initial phase of the disease trajectory; (2) hope and information further into the disease trajectory and (3) personal, professional and organisational factors that influenced information and communication throughout the disease trajectory.

Conclusion The participants' experience of being told for the first time that they had an incurable disease was perceived as inadequate, while postsurgery palliative chemotherapy, physicians and nurses offered hope. The participants preferred customised information about their treatment and likely future prospects and physicians and nurses who took a holistic and compassionate approach focusing on their lifeworld. To be a sensitive, holistic and compassionate physician or nurse requires knowledge and confidence. To achieve this requires training and guidance at universities and in hospitals.

\section{BACKGROUND}

Patients with cancer who are treated with palliative intent receive extensive amounts of disease-related information from the first
Strengths and limitations of this study

- In-depth and rich knowledge derived from the thoughts of 20 patients undergoing palliative care for colorectal cancer about how information about their disease, prognosis and life expectancy was communicated, starting from the first time that they were told that they had an incurable disease through to their postsurgery treatment.

- The qualitative design revealed that patients with colorectal cancer undergoing palliative care prefer health care professionals who are compassionate at all stages of their disease trajectory.

- It could be seen as a limitation that the study focused on one group of patients in palliative care, because this could limit the variation in findings that might have been evident with inclusion of more heterogeneous groups.

- We interviewed the patients at only one time point during chemotherapy and their memory about receiving their first information relating to their disease may have been coloured by later experiences.

time they are informed about the incurable nature of their disease through the following months or years of treatment and care. ${ }^{1-4}$ Guidelines encourage healthcare professionals (HCPs) such as physicians and nurses to keep patients informed and to discuss their prognoses and likely future prospects. However, many HCPs and patients struggle to find the right approach for these discussions, ${ }^{5-9}$ and a primary focus on open communication regarding the bleak prospects for the patient's life expectancy entails a risk of overwhelming the individual's need for information and their hope. ${ }^{10}$ In-depth studies of patients' experiences about information given by physicians and nurses throughout their disease trajectory are needed to guide HCPs in how to communicate to patients 
undergoing palliative care information about their diagnosis and life expectancy.

Most studies focusing on patient-HCP communications about disease and prognosis in patients with incurable cancer are quantitative and involve patients at either an early or late stage of the disease. ${ }^{611}$ Qualitative studies report divergent results regarding the patient's acceptance of the chronic and incurable nature of their disease and the presentation of their prognosis. ${ }^{11-17}$ Patients request that both disease-oriented and illness-oriented information be provided by caring and trusted HCPs. ${ }^{13} 1417$

Patients with cancer undergoing palliative treatment are vulnerable, and good patient-HCP relationships are important. ${ }^{18}$ Løgstrup ${ }^{19}$ emphasised the importance of trust and the patients' vulnerability in such relationships, while Mishler ${ }^{20}$ distinguished between the voice of medicine (the technical-scientific assumptions of medicine) and the voice of the lifeworld (the natural attitudes of everyday life) in patient-physician communication. Mishler suggested an increased attentiveness to the voice of the patients in terms of their lifeworld, especially in vulnerable individuals such as patients in palliative care.

Patients often experience a life crisis when they are informed that their cancer is incurable. ${ }^{21}$ Over time, the majority adjust to their new life situation, and during this time, their preferences and experiences regarding information and communication might change. ${ }^{18}$ Although colorectal cancer is one of the most common types of cancer, ${ }^{21}{ }^{22}$ there is limited knowledge about how this patient group views information and communication about disease and life expectancy throughout their disease trajectory, because most studies include heterogeneous groups of patients. Treatment for colorectal cancer usually involves surgical removal of the tumour followed by adjuvant chemotherapy. Thus, most patients with colorectal cancer tend to have a similar disease trajectory, and knowledge about their experience and information preferences might be valuable to give patients better palliative care.

We aimed to explore the experiences of patients with incurable colorectal cancer and their reflections on information provided by physicians and nurses while they were in palliative care. Our main focus was the patients' thoughts about how information about their disease, prognosis and life expectancy was communicated, from the first time that they were told that their disease was incurable through to postsurgery palliative treatment.

\section{METHODS}

We chose a qualitative inductive approach using in-depth interviews. ${ }^{23}$ As part of a larger study, ${ }^{24}$ we invited patients with metastatic colorectal cancer who were referred for palliative chemotherapy at three regional hospitals in Southern Norway to participate in this study. Oncologists informed patients about the study at the outpatient clinics when they attended for the second or third cycle of chemotherapy. Most participants were informed of their incurable diagnosis by surgeons, except for two who were informed by their general practitioners (GPs). All participants had undergone surgery for their cancer, and most had their surgery at relatively small hospitals, with surgeons being mainly responsible for the patients' care and the communication in this phase. The participants spent only a few days in the surgery department with teams including few HCPs. Subsequently, chemotherapy was provided at an oncological outpatient clinic where oncologists were mainly responsible for the treatment. The participants visited the outpatient clinic for weeks or months.

The patients were eligible for inclusion if they were aged 18 years or older, had metastatic colorectal cancer, had undergone surgery for their cancer, had been referred for first-line or second-line palliative chemotherapy, had a life expectancy of $>6$ months and were able to give written informed consent. We included consecutive patients of different ages and marital status and with varying demographic and clinical characteristics. ${ }^{25} \mathrm{We}$ excluded patients with any significant comorbidity that could compromise their life expectancy, or who were unable to understand or read Norwegian. Patients with conditions that the physician believed could affect the patient's ability to understand or cope with the questions were considered ineligible, including patients who were considered to be too emotionally vulnerable $(n=4)$.

Twenty patients with colorectal cancer (34-75 years of age) were invited to participate in the study over a period of 1 year, and all accepted the invitation. All patients received combination chemotherapy (table 1 ) and had few physical symptoms related to their disease. The sample comprised 12 patients receiving first-line chemotherapy (five women and seven men) and eight receiving second-line chemotherapy (three women and five men).

Table 1 Characteristics of patients receiving non-curative chemotherapy

\begin{tabular}{lll}
\hline & First line $(\mathbf{n = 1 2})$ & $\begin{array}{l}\text { Second line } \\
(\mathbf{n}=8)\end{array}$ \\
\hline Women & 5 & 3 \\
Men & 7 & 5 \\
\hline $\begin{array}{l}\text { Mean age (range), years } \\
\text { Marital status: }\end{array}$ & $63(34-75)$ & $69(64-75)$ \\
\hline $\begin{array}{l}\text { Married/cohabiting } \\
\text { Single }\end{array}$ & 10 & 8 \\
\hline $\begin{array}{l}\text { Widow/widower } \\
\text { Chemotherapy used }\end{array}$ & 1 & \\
$\quad$ Fliri/bevacizumab & 1 & \\
$\begin{array}{l}\text { FLOX (5-fluorouracil, folinic } \\
\text { acid, oxaliplatin) }\end{array}$ & 1 & 8 \\
$\begin{array}{l}\text { Capecitabine plus } \\
\text { oxaliplatin (Xelox) }\end{array}$ & 10 & \\
\hline
\end{tabular}

All paticipants received 5-fluorouracil-based combination chemotherapy with irinotecan or oxaliplatin, \pm bevacizumab. 
Eleven patients were treated by oncologists, and nine were treated by junior physicians.

\section{Data collection}

The same researcher (GR) conducted all the interviews. One interview took place at the patient's home, and the other interviews took place at the cancer centre or outpatient clinics at a time when the patients had an appointment. The researchers did not know the patients before the interviews and did not treat the patients. The meetings were in-depth interviews lasting 50-100 min using a semistructured interview guide to ensure inclusion of the issues in focus, ${ }^{23}$ and questions such as: 'What do you think about the first information that you received about your disease and its prognosis?', 'How was information provided about the follow-up chemotherapy and likely future prospects?', 'Have you received the information that you expected or is there anything missing?' and 'What things are important when giving information about your disease and prognosis, and how do you want it to be given/delivered?' After conducting 11 interviews, we did some preliminary analyses and made minor changes to the interview guide to obtain more data on issues that needed to be expanded to address the research aim; for example, 'What characterised the good information that you received versus other information that you were not happy with?' Patients were included until data saturation was achieved, as indicated by only minor new information being obtained in interviews 19 and $20{ }^{23}$ At $2-4$ days after each interview, GR contacted the patient and asked whether the interview had influenced him or her negatively. No patient experienced a negative influence or reaction.

\section{Analysis}

We audiotaped and transcribed the interviews verbatim and made logs after each interview. The data were analysed by qualitative content analysis to identify the themes in the data. For the analyses, we (1) read all the interviews to understand the meaning of the whole text, (2) investigated sentences or sections to clarify their meaning and to facilitate the identification of themes, (3) related sentences or sections to the meaning of the whole text and (4) identified passages representative of shared understanding between the researchers and participants. To support the analysis, we created mind maps and discussed the analysis. The analysis steps were followed carefully, which increased the reliability of the study. Quotations are used to illustrate and support the findings, which increases their trustworthiness. To validate the findings, all authors participated in discussions of the empirical analysis and in writing up the findings. In the discussion, the findings were interpreted in light of our previous understanding. GR and US are both nurses and professors in health sciences with clinical experience in palliative care. IV is a gynaecologist and professor who also has extensive experience in treating patients with cancer who are undergoing palliative care.
Voluntariness and confidentiality were assured during the collection, handling and reporting of data. ${ }^{26} 27$

\section{Patient involvement}

Before we started the study, we conducted three pilot interviews with patients with cancer to test the study design and the interview guide, and made minor changes to the guide. These interviews were not included in the study. There was no further patients' involvement. The findings are given in this publication.

\section{Results}

Through data-driven empirical analysis, we identified three themes: (1) inadequate information during the initial phase of the disease trajectory; (2) hope and information further into the disease trajectory and (3) personal, professional and organisational factors that influenced information and communication throughout the disease trajectory. We did not identify any differences between participants receiving first-line or second-line chemotherapy.

\section{Inadequate information during the initial phase of the disease trajectory}

The news that their cancer was incurable was given to patients at the surgical department or by the patient's GP. Overall, how patients experienced receiving this information varied: it could have been given earlier, it was experienced as a shock, it was insufficient, it was given in an inappropriate way or at an inappropriate place. However, some reported that they were satisfied with the way the information was given.

A few participants had to wait a long time (weeks or months) from their first concern about the disease until they were examined or had an appointment at the hospital. When the cancer was finally diagnosed, they received limited apologies for the delay from the physicians, and emphasised that an apology would have made the situation easier to handle. Some had not even felt particularly ill, and it was hard for them to understand the message from the physician that they had an incurable disease. Several participants experienced the first information about the incurable nature of their disease as a shock.

When the surgeon gave me the message that my disease was incurable, I was shocked, I didn't feel that anything was wrong. I asked him how long I had left to live. He just shrugged and didn't have any answer. The conversation took $8 \mathrm{~min}$ (patient 4 , woman aged 54 years).

We did not identify any difference between the two participants who received the news from their GP compared with those who received it from their surgeon.

Some participants felt that the information given before and after their surgery was insufficient. The information was brief, there was no time after the surgery for further communication, and a few participants felt that 
the HCPs had not told them the whole truth. They would have liked more answers and sufficient communication with the surgeon.

I think she gave the message in three sentences. She said I had metastatic cancer. That's it. I asked what it meant. 'I don't know' she replied (patient 6, man 73 years).

In contrast, some of the participants, males in particular, expressed satisfaction with how the surgeon had given preoperative and postoperative information and explained the surgery, its consequences and likely future treatment-related effects, for example, challenges with the stoma or the risk of impotence after the operation.

I was happy with the information the surgeon gave. I am a person who asks questions, and I am not afraid of asking. I received the answers I needed (patient 15, man aged 73 years).

A few participants reported that surgeons or GPs had given them the news in an inappropriate way or at an inappropriate place (eg, in a small examination room) and they experienced this as an extra burden. Further questions from the participants were answered to a limited extent, if at all. It was challenging to be told that their cancer could not be cured. A few participants received the message that a complete tumour resection was impossible or that very little could be done. Such messages were experienced as a death sentence.

It's important to tell the truth, but in an appropriate way. 'Go home and die'. That's not appropriate (patient 4 , woman aged 54 years).

Although the message was brutal to hear, a few participants admitted that a straightforward message was probably the best way.

\section{Hope and information further into the disease trajectory}

Postsurgery chemotherapy and further information and care were offered/given at the cancer centre. Hope was offered by the palliative chemotherapy itself, as well as by physicians and nurses, and there was variation in how much and how precise information the participants preferred in this phase.

When the participants started their postsurgery chemotherapy, some time had passed and further treatment implied hope that something could be done after all. The behaviour and attitudes of physicians and nurses also offered hope. At the cancer centre, the participants were met with openness, knowledge and sufficient time. The participants experienced that the physicians postponed death by offering chemotherapy, and the importance of including hope in patient communication was emphasised.

She asked about my background, she saw more than my illness. She looks at you. She gives you hope. That is how I want to be met (Patient 4, woman aged 54 years).

The participants' hopes seemed to change from before they were diagnosed with their incurable disease and through their disease trajectory. Physicians and nurses at the cancer centre conveyed that they would try to delay disease progress and relieve pain and symptoms. Even though they recognised that their cancer was incurable, most participants hoped that they would be among those who could live for years despite a poor prognosis. As the disease progressed, they hoped for good days, not extraordinary things or experiences, and for some participants there seemed to be a change in goals and values.

Correct and truthful information about their disease, treatment effects, side effects, metastases and likely future prospects was important for the participants. Preferences varied regarding the amount of information they wanted to receive and at which time point. Some participants wanted a total overview of their disease and prognosis from the start, some wanted a smaller amount of information at that time, while others wanted their body to tell them how their disease was progressing.

I don't want to know the exact date. I would like information about disease progression and prognosis bit by bit, or let my body tell me bit by bit (patient 17, woman aged 71 years).

Some participants found vague information about likely prospects confusing. In particular, some of the male participants wanted straightforward information.

I would like to know even more if it is possible. I don't want them to keep any information back. I would like to have a better overview and know what to expect in the future (patient 13, man aged 68 years).

During palliative chemotherapy, the participants had different experiences of receiving information about their life expectancy. Some found the information to be adequate, while others claimed that they had received non-specific information on this topic, if anything at all.

They haven't said much about life expectancy. However, the treatment is palliative. They haven't given me the time. And I haven't asked (patient 7, man aged 63 years).

\section{Personal, professional and organisational factors that influenced information and communication throughout the disease trajectory}

Throughout their disease trajectory, the participants had experiences and preferences relating to personal, professional and organisational factors that influenced information and communication. The participants experienced that in the surgery department, there was limited time for information and communication. They preferred HCPs who were knowledgeable and took a holistic approach, 
and that their healthcare is organised in such a way that it was possible to meet the same well-qualified HCPs.

Most participants met the surgeon once before the surgery and spent only a few days in the surgery department. Some experienced that there was too little time for information and communication.

I only received a small amount of information at the surgical department. They just sent me home. You're finished, you can leave. I would have liked more answers (patient 20, man 74 years).

The combination of the professional knowledge and personality of the HCPs was emphasised as important. The participants strongly appreciated physicians and nurses who had enough time for them and who knew them and their disease. One participant characterised this as follows.

She is an oncologist with a heart and a brain (patient 4 , woman 54 years).

The participants wanted physicians and nurses who could see them as a person, not just as a patient. They emphasised the importance of the HCP paying attention, making them feel that there was time enough for discussions during the consultations or visits at the cancer centre for chemotherapy and knowing them without consulting the computer record.

He saw the person. It was the warmth in his eyes and the way that he sat relaxed in his chair. I don't remember much from the consultation. I just remember the feeling (patient 18, woman aged 34 years).

The participants also wanted to see physicians and nurses who took a holistic approach to treatment and care, who took part in their lifeworld, not just the physical and mechanical components related to their disease; in other words, they wanted a compassionate physician or nurse. Indeed, the characteristics of the best physicians or nurses were emphasised as knowledge, warmth and trust, because such qualities were important for how the participants felt, for their hopes and for how they dealt with their disease.

When I come to the cancer centre, I feel it's about me. I know there are hundreds of people coming and going. But every time I come, I feel that they are taking care of me (patient 6 , man 72 years)

The participants preferred to be treated by knowledgeable HCPs who were able to answer questions. Some felt insecure if they were treated by a junior physician who could not answer all their questions.

I would have felt more secure if I was treated by a specialist, one who didn't have to ask colleagues to be sure. At least occasionally (patient 11, man aged 60 years).

Furthermore, the participants preferred to receive their test results immediately rather than to wait until their next appointment at the outpatient clinic or the cancer centre.

There were minor changes after the last computer tomography. The oncologist telephoned and told me the results. I didn't have to wait for the next appointment, I didn't have to worry until then (patient 17, woman aged 71 years).

Most participants wanted their healthcare and treatment to be organised in such a way that it was possible to see the same physician at each consultation, and they appreciated small units/departments. The participants appreciated the possibility of contacting the physicians and nurses if needed, to have 'an open door'. Some of those who had to alternate between different physicians felt that they had to start from the beginning each time, which they found exhausting.

I am an introverted person. I am not able to speak openly with everyone. When I meet a new physician, I have to start from the beginning, and I don't like it. And it's OK to feel like this. We're all different (patient 2 , woman aged 73 years).

In addition to the discomfiting feeling of having to deal with new physicians, some participants reported that information was not forwarded between the different physicians, resulting in misunderstandings. They felt insecure and that no one was in charge of their medical care, for example, when experiencing changes in treatment at the same time as they changed physicians/junior doctors. Further, some participants highlighted that a lack of coordination in treatment and care imposed an extra burden, and emphasised that confidence was inspired by better organisation and by the professional knowledge and ability of nurses and physicians to answer questions.

\section{DISCUSSION}

To our knowledge, this is the first study to explore the thoughts of patients with colorectal cancer undergoing palliative care about how information was communicated about their disease, prognosis and life expectancy, from the first time that they were informed that their disease was incurable to postsurgery palliative treatment.

Evident in our findings was the asymmetrical relationship in which the physicians held the knowledge and expertise about the disease and the participants had to trust them. As Løgstrup ${ }^{19}$ emphasises, trust is something fundamental to our lives and implies that you expose yourself to others and become vulnerable. Vulnerability implies that others are in control and hold their fellow humans' lives in their hands. The responsibility of HCPs in such asymmetrical relationships is especially important in palliative care. The participants wanted information about their incurable cancer to be provided in a sensible and sensitive way, in a setting that allowed enough time. However, according to many of our participants, the communication in these meetings failed to give them 
sufficient help to deal with the information and their vulnerability. Being the first to inform patients that they have an incurable disease is difficult, and bearers of bad news may later be blamed despite their best intentions to provide information in a sensitive manner. Furthermore, in surgical departments, there is limited time allocated for surgeon-patient communication, and doctors in surgical specialties are significantly more likely to be rated poorly than non-surgical specialists or GPs when breaking bad news. ${ }^{18}$

Previous studies highlight that palliative treatment implies hope. Hope is an important coping strategy in such patients, ${ }^{28}{ }^{29}$ and has been described as essential for human life. The realistic hope for most of our participants was that something could be done to relieve their symptoms and potentially to postpone death, and to enable them to lead ordinary everyday lives and have the possibility of spending time with family and friends. Therefore, the participants emphasised the importance of HCPs including hope in their communications of disease, prognosis and life expectancy throughout the disease trajectory. Studies have shown that there is a fine balance between telling the truth and nurturing hope, and that there is a spectrum of hope, from hope for a cure to hope for living as normally as possible. ${ }^{1630}$ This aspect was also identified in our study.

There was diversity in how detailed the participants wanted information about their disease and likely future prospects to be. Previous work indicates that patients with incurable cancer want truthful information about their disease, treatment and likely future prospects. ${ }^{31} 32$ However, because of individual preferences, individually customised approaches would seem desirable, ${ }^{33} 34$ and could be considered an important part of a patient's lifeworld that should be attended to in communication between patients and physicians or nurses. Although all the participants in the present study were aware of the incurable nature of their disease, we did not explore the accuracy of their prognostic awareness. However, in a systematic review and meta-regression analysis, Chen et $a l^{35}$ identified that only half the patients with cancer with advanced disease accurately understood their prognosis.

In our study, the participants emphasised the importance of organising all their palliative treatment and care with well-qualified physician or nurse. They seemed to prefer that physician or nurse communications included what Mishler ${ }^{20}$ has characterised as the "voice of medicine', which mainly focuses on the symptoms and medical and technical problems or aspects of the disease. But they also wanted physicians and nurses to initiate communication focusing on the participants' inner thoughts related to their illness, $\mathrm{Me}^{20}$ 'voice of the lifeworld', which included asking more open-ended questions. Physicians and nurses who do this are characterised as compassionate caregivers. ${ }^{36}$

\section{Implications for healthcare}

It might be considered overly demanding to be the bearer of the bad news that a patient has an incurable disease. Some of our participants experienced the first information about their incurable disease as delayed, insufficient, given in an inappropriate way or at an inappropriate place. This indicates that there is a need for increased focus on communication by HCPs both during their university studies and in hospitals. ${ }^{8}$ Furthermore, it would be desirable for surgeons to have more time allocated to conveying information and communicating with patients.

Physicians and nurses have extensive responsibilities in how they communicate with patients who have an incurable disease, particularly because of the asymmetrical relationship between patients and HCPs. The HCPs have knowledge about how the disease will most likely progress and about common psychological responses. However, the patients' inner thoughts and lifeworld are not necessarily known to the HCPs. The responsibility to invite or initiate communication about the patients' inner thoughts is in the hands of the physicians and nurses. Furthermore, it is important to strive for a more symmetrical relationship between patients and HCPs, ${ }^{19} 28$ which will also increase the possibility of shared decision-making in treatment and care.

The participants preferred compassionate physicians and nurses. Being compassionate requires more than empathy; it requires knowledge, proactivity and interconnectedness. ${ }^{36}$ Furthermore, to become a compassionate physician or nurse, training is required involving observation, guidance and feedback about one's own practice. ${ }^{36}$ HCPs also need to be aware of how much information each patient prefers and discuss this with the patient. Previous studies have shown that this awareness is associated with years of practice and confidence. ${ }^{810}$ Treatment and care of patients undergoing palliative chemotherapy should be organised in such a way that patients are able to see the same well-qualified physician and optionally also the same nurses at each consultation. Furthermore, palliative healthcare should include guidelines on how to treat the patients more smoothly, and allow enough time for communication with this vulnerable patient group.

\section{Methodological considerations}

The strengths of the study are that the 20 participants provided us with rich data about their experiences, feelings and reflections on the information and communication by HCPs about their disease and life expectancy during their disease trajectory. Qualitative content analysis aims to stay close to the data and texts to elucidate the findings, although our preunderstanding of the issues as researchers might also have influenced the analysis of the data. Another strength of our study is that it included patients with one type of cancer who were in the palliative phase. Colorectal cancer is the second most common cancer diagnosed in women worldwide, and the third most common cancer diagnosed in men ${ }^{21}{ }^{22}$; thus, the knowledge gained in this study could be applied to this 
large group of patients. However, it is also possible that studying just one patient group might limit the variation in findings that may have been identified by including more heterogeneous groups. We have limited systematic information about the participants' sociodemographic variables, common behaviour and coping mechanisms that might have influenced their experiences and preferences. However, based on the few characteristics, we identified during the interviews, the variations in sociodemographic factors such as gender, age and marital status seem to be similar to those of patients with colorectal cancer reported by Jemal et $_{\text {al. }}{ }^{21}$ Although our findings are not generalisable to patients with other cancer diagnoses, they may be transferable to hospitals with a similar organisation of surgery and postsurgery palliative treatments.

\section{CONCLUSIONS}

The findings of this study provide a deeper understanding about how patients with incurable colorectal cancer undergoing palliative treatment experience and reflect on HCP-patient communication about disease and life expectancy from before surgery through to postsurgery chemotherapy. The process of receiving the first information that they had an incurable disease was generally experienced as inadequate, while postsurgery palliative chemotherapy, physicians and nurses offered hope. The participants preferred customised information about treatment and likely future prospects, and physicians and nurses who used a holistic approach focusing on their lifeworld with compassion. To become a sensitive, holistic and compassionate physician or nurse requires knowledge and confidence, and to achieve this, training and guidance are needed.

Acknowledgements We thank the Department of Clinical Research at Sorlandet Hospital and the Faculty of Health and Sport Science, University of Agder for funding the study. GR was a visiting researcher, as an affiliate academic, in the Marie Curie Palliative Care Research Department, University College London, January to June 2017 while writing most of the paper. We wish to thank the three patients who helped us with pilot interviews during the design of the study, as well as all the patients who participated.

Contributors GR and IV were responsible for the study design. GR was responsible for the patient interviews and data collection. GR, US and IV contributed to a critical appraisal of the analysis, manuscript preparation and read and approved the final version of the manuscript.

Funding The authors have not declared a specific grant for this research from any funding agency in the public, commercial or not-for-profit sectors.

Competing interests None declared.

Patient consent for publication Obtained.

Ethics approval The study was approved by the Regional Committee for Medical Research Ethics (REK Southeast 2011/2464).

Provenance and peer review Not commissioned; externally peer reviewed.

Data sharing statement Due toconfidentiality and the need to protect patient identity, it is not possible toshare the raw data

Open access This is an open access article distributed in accordance with the Creative Commons Attribution Non Commercial (CC BY-NC 4.0) license, which permits others to distribute, remix, adapt, build upon this work non-commercially, and license their derivative works on different terms, provided the original work is properly cited, appropriate credit is given, any changes made indicated, and the use is non-commercial. See: http://creativecommons.org/licenses/by-nc/4.0/.

\section{REFERENCES}

1. McRee AJ, Goldberg RM. Optimal management of metastatic colorectal cancer: current status. Drugs 2011;71:869-84.

2. Cameron J, Waterworth S. Patients' experiences of ongoing palliative chemotherapy for metastatic colorectal cancer: a qualitative study. Int J Palliat Nurs 2014;20:218-24.

3. Cameron MG, Kersten C, Guren MG, et al. Palliative pelvic radiotherapy of symptomatic incurable prostate cancer - a systematic review. Radiother Oncol 2014;110:55-60.

4. Cameron MG, Kersten C, Vistad I, et al. Palliative pelvic radiotherapy for symptomatic rectal cancer - a prospective multicenter study. Acta Oncol 2016;55:1400-7.

5. Hancock K, Clayton JM, Parker SM, et al. Truth-telling in discussing prognosis in advanced life-limiting illnesses: a systematic review. Palliat Med 2007;21:507-17.

6. Hagerty RG, Butow PN, Ellis PM, et al. Communicating prognosis in cancer care: a systematic review of the literature. Ann Oncol 2005;16:1005-53.

7. Chou WS, Hamel LM, Thai CL, et al. Discussing prognosis and treatment goals with patients with advanced cancer: a qualitative analysis of oncologists' language. Health Expect 2017;20:1073-80.

8. Suwanabol PA, Kanters AE, Reichstein AC, et al. Characterizing the Role of U.S. Surgeons in the provision of palliative care: a systematic review and mixed-methods meta-synthesis. J Pain Symptom Manage 2018;55:1196-215.

9. Dillon BR, Healy MA, Lee CW, et al. Surgeon perspectives regarding death and dying. J Palliat Med 2019;22:132-7.

10. Rogg L, Aasland OG, Graugaard PK, et al. Direct communication, the unquestionable ideal? Oncologists' accounts of communication of bleak prognoses. Psychooncology 2010;19:1221-8.

11. Murray CD, McDonald C, Atkin $\mathrm{H}$. The communication experiences of patients with palliative care needs: a systematic review and meta-synthesis of qualitative findings. Palliat Support Care 2015;13:369-83.

12. Martinsson L, Axelsson B, Melin-Johansson C. Patients' perception of information from physicians during palliative chemotherapy: a qualitative study. Psychooncology 2016;14:495-502.

13. Kirk P, Kirk I, Kristjanson LJ. What do patients receiving palliative care for cancer and their families want to be told? A Canadian and Australian qualitative study. BMJ 2004;328:1343.

14. Butow PN, Dowsett $S$, Hagerty $R$, et al. Communicating prognosis to patients with metastatic disease: what do they really want to know? Support Care Cancer 2002;10:161-8.

15. Clarke MG, Kennedy KP, MacDonagh RP. Discussing life expectancy with surgical patients: do patients want to know and how should this information be delivered? BMC Med Inform Decis Mak 2008;8:24.

16. Clayton JM, Butow PN, Arnold RM, et al. Fostering coping and nurturing hope when discussing the future with terminally ill cancer patients and their caregivers. Cancer 2005;103:1965-75.

17. Stajduhar KI, Thorne SE, McGuinness L, et al. Patient perceptions of helpful communication in the context of advanced cancer. J Clin Nurs 2010;19:2039-47.

18. Barnett MM. Effect of breaking bad news on patients' perceptions of doctors. J R Soc Med 2002;95:343-7.

19. Løgstrup KE. The ethical demand. Notre Dame, III: University of Notre Dame Press, 1997.

20. Mishler G. The discourse of medicine - dialectics of medical interviews. New Jersey: Ablex Publishing Corporation, Norwood, New Jersey, 1984.

21. Jemal A, Center MM, DeSantis C, et al. Global patterns of cancer incidence and mortality rates and trends. Cancer Epidemiol Biomarkers Prev 2010;19:1893-907.

22. Torre LA, Bray F, Siegel RL, et al. Global cancer statistics, 2012. CA Cancer J Clin 2015;65:87-108.

23. Crabtree BF, Miller WL. Doing qualitative research. Thousand Oaks, Calif: Sage, 1999.

24. Rohde G, Kersten C, Vistad I, et al. Spiritual well-being in patients with metastatic colorectal cancer receiving noncurative chemotherapy: a qualitative study. Cancer Nurs 2017;40:209-16.

25. Polit DF. Essentials of nursing research: methods, appraisal, and utilization. Philadelphia: Lippincott Williams and Wilkins, 2006.

26. Beauchamp TL, Childress JF. Principles of biomedical ethics. 7th edn. New York: Oxford University Press, 2013.

27. WMA Declaration of Helsinki. Ethical principles for medical research involving human subjects. 2013 http://www.wma.net/en/ 30publications/10policies/b3/ 
28. Hegarty M. The dynamic of hope: hoping in the face of death. Prog Palliat Care 2001;9:42-6.

29. Rustøen T. Hope and quality of life, two central issues for cancer patients: a theoretical analysis. Cancer Nurs 1995;18:355-61.

30. Clayton JM, Butow PN, Arnold RM, et al. Discussing life expectancy with terminally ill cancer patients and their carers: a qualitative study. Support Care Cancer 2005;13:733-42.

31. Miccinesi G, Bianchi E, Brunelli C, et al. End-of-life preferences in advanced cancer patients willing to discuss issues surrounding their terminal condition. Eur J Cancer Care 2012;21:623-33.

32. Hagerty RG, Butow PN, Ellis PA, et al. Cancer patient preferences for communication of prognosis in the metastatic setting. J Clin Oncol 2004;22:1721-30.
33. Mackenzie LJ, Carey ML, Paul CL, et al. Do we get it right? Radiation oncology outpatients' perceptions of the patient centredness of life expectancy disclosure. Psychooncology 2013;22:2720-8.

34. Walczak A, Butow PN, Davidson PM, et al. Patient perspectives regarding communication about prognosis and end-of-life issues: how can it be optimised? Patient Educ Couns 2013;90:307-14.

35. Chen $\mathrm{CH}$, Kuo SC, Tang ST. Current status of accurate prognostic awareness in advanced/terminally ill cancer patients: Systematic review and meta-regression analysis. Palliat Med 2017;31:406-18.

36. Larkin PJ. Compassion - The essence of palliative and end-of-life care. 1st edn. New York: Oxford University Press, 2016. 